\title{
THE OESOPHAGUS LINED WITH GASTRIC MUCOUS MEMBRANE
}

\author{
BY \\ P. R. ALLISON AND A. S. JOHNSTONE \\ Leeds
}

(RECEIVED FOR PUBLICATION FEBRUARY 26, 1953)

Peptic oesophagitis and peptic ulceration of the squamous epithelium of the oesophagus are secondary to regurgitation of digestive juices, are most commonly found in those patients where the competence of the cardia has been lost through herniation of the stomach into the mediastinum, and have been aptly named by Barrett (1950) " reflux oesophagitis." In the past there has been some discussion about gastric heterotopia as a cause of peptic ulcer of the oesophagus, but this point was very largely settled when the term reflux oesophagitis was coined. It describes accurately in two words the pathology and aetiology of a condition which is a common cause of digestive disorder. In the same paper Barrett for the first time drew a sharp distinction between the above and lesions which had previously been described by pathologists as peptic ulcers of the oesophagus, but which in fact were gastric ulcers occurring in gastric mucous membrane lining a viscus which, from the outside, looked like oesophagus.

In retrospect it is now easy to see how the confusion had arisen. Peptic ulcer of the oesophagus is associated with dense submucous fibrosis. The ulcer itself tends to remain superficial; it may ooze blood, but rarely causes severe and sudden bleeding and does not perforate. It causes fibrous stenosis. Patients with this condition may die at home, but if they come to necropsy the stricture may be missed, the hernia is almost certainly missed, and the lesion, if found, is described as a simple stricture. There is little that seems worthy of preservation in a museum, especially when the lesion is made even more insignificant by severe post-mortem digestion. When a part of the oesophagus is lined with gastric mucous membrane, and a gastric ulcer occurs within this area, it behaves, as Barrett has shown, like an ulcer in the abdominal stomach; it may cause stenosis, it may bleed profusely, and it may perforate. A lesion such as this that perforates into the pleura immediately attracts the attention of the pathologist, and is likely to find its way into the museum. The result has been that pathologists have been describing one thing and clinicians another, and they have had the same name. The clarification of this point has been so important, and the description of a gastric ulcer in the oesophagus so confusing, that it would seem to be justifiable to refer to the latter as Barrett's ulcer. The use of the eponym does not imply agreement with Barrett's description of an oesophagus lined with gastric mucous membrane as "stomach." Such a usage merely replaces one confusion by another. All would agree that the muscular tube extending from the pharynx downwards and lined with squamous epithelium may be correctly referred to as the oesophagus or gullet. The dilated sac covered with peritoneum and lined with gastric mucous membrane is obviously stomach whether it lies in the abdomen or is herniated into the mediastinum. A glance at specimens of congenital oesophageal atresia shows that the lower segment of the oesophagus is developed in close association with the stomach, and there may therefore be some embryological support for calling it stomach when it is lined with gastric mucous membrane. But more careful examination of such a specimen shows that it has no peritoneal covering, that the musculature is that of the normal oesophagus, that there may be islands of squamous epithelium within it, that there are no oxyntic cells in the mucosa, and that in addition to gastric glands there are present typical oesophageal mucous glands. Such a congenital abnormality has been associated in the present series and in other reported specimens with a sliding hernia of the true stomach.

If an oesophagus lined with gastric mucosa is to be called stomach it leads to an impasse in description, especially if the true stomach is also in the mediastinum. Further than this, such a nomenclature can lead to a serious error in interpretation and to incorrect operative treatment, as will be shown in the present series of patients. It appears better, therefore, to refer to that congenital abnormality 


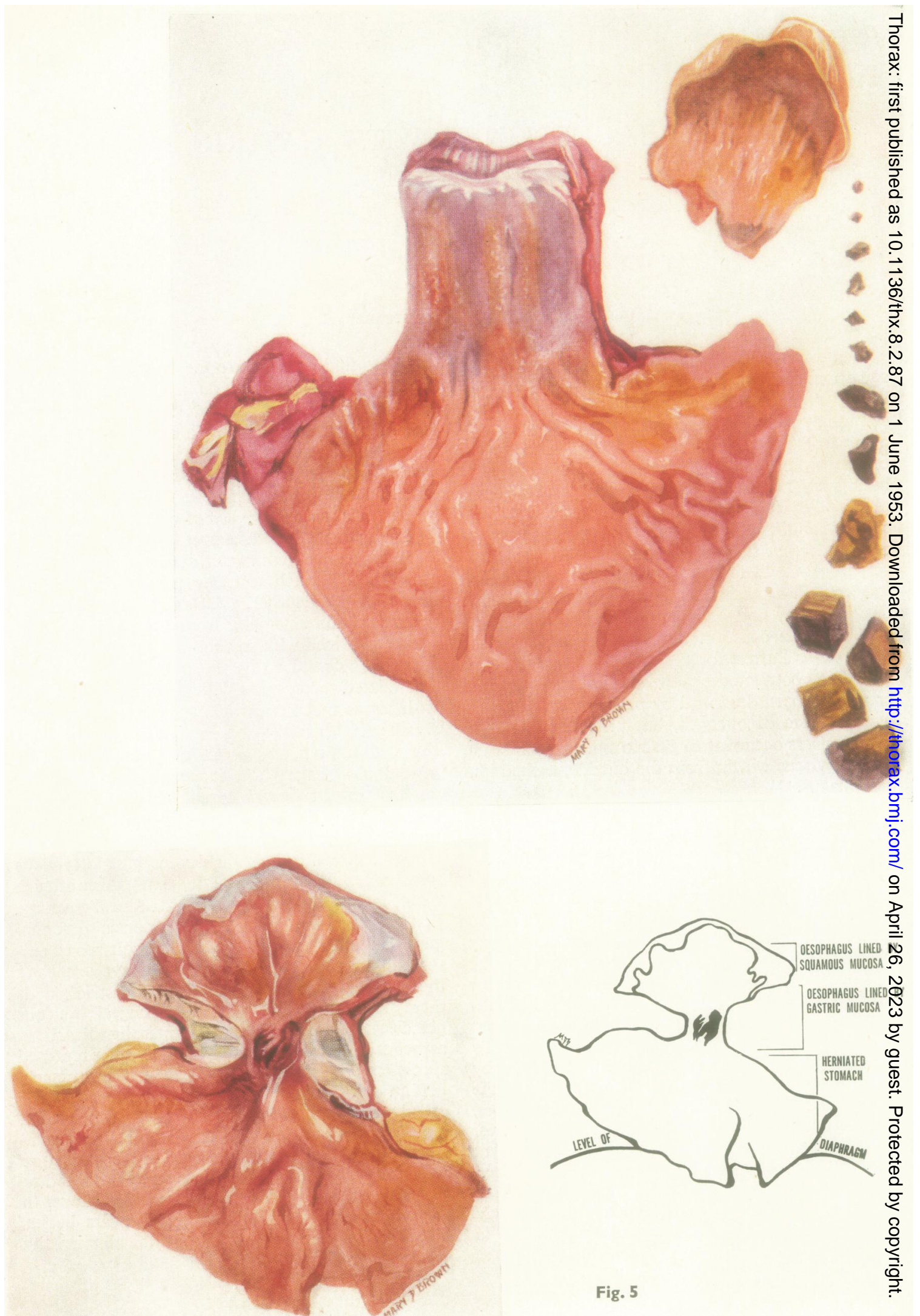


Fig. 1.-Resected lower oesophagus and herniated portion of stomach from Case 1 . The narrow strip of squamous lining at the top of the oesophagus is not enough to show the oesophagitis. The line drawing represents the various segments in relation to the diaphragm.

FIG. 2.-Radiograph of Case 2 showing the picture of three segments. The top segment is lined by squamous epithelium. Just below the aortic arch is a stricture from reflux oesophagitis. This separates the first from the second segment, the latter being ossophagus lined by gastric mucous membrane. The constriction caused by the cardia marks the top of the third segment, which is stomach herniated above the diaphragm.
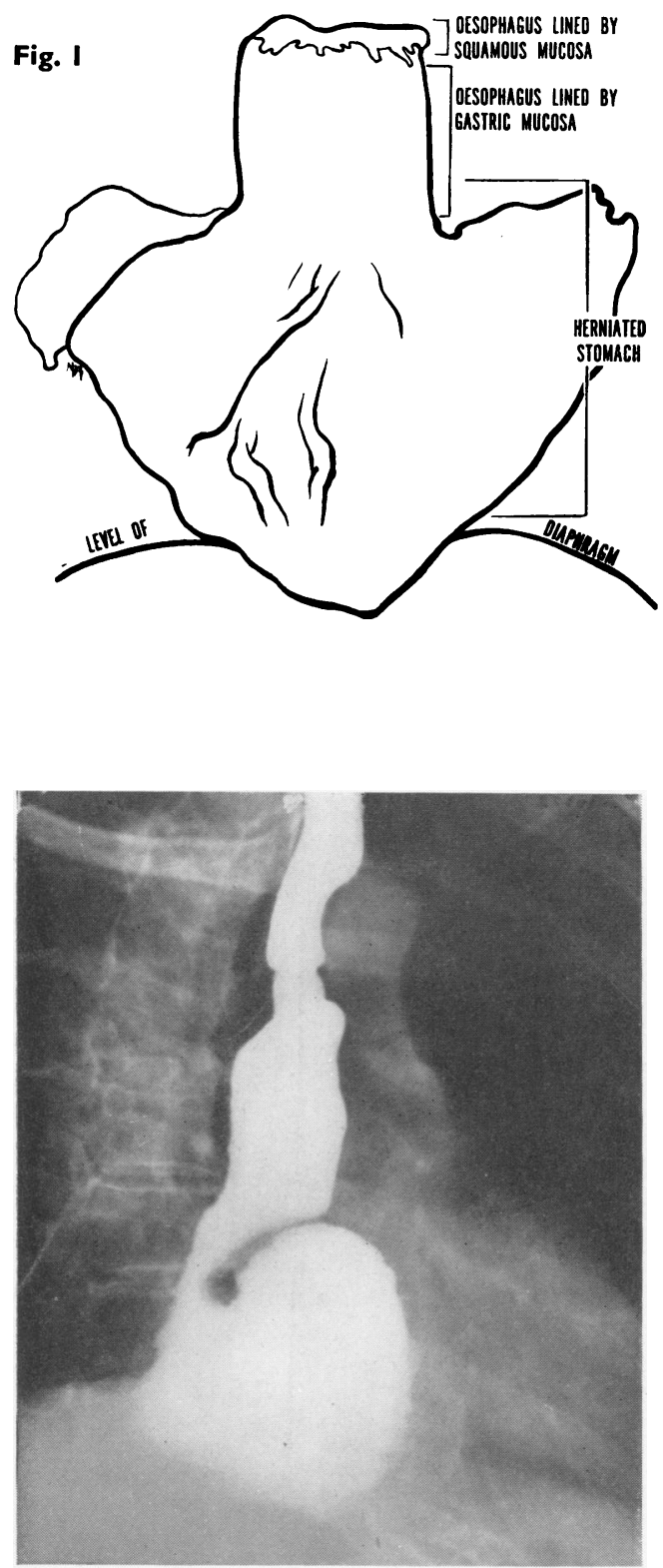

Fig. 2
Fig. 3.-Radiograph of Case 3 showing the three segments as in Fig. 2.

FIG 4.-Radiograph of Case 4 showing an irregular stenosis from a gastric ulcer of the oesophagus with a sliding hernia of true stomach.

FIG. 5.-Excised specimen from Case 4: a typical stenosing, penetrating Barrett's ulcer. Only a small part of the true herniated stomach is included in the picture, the rest being oesophagus lined by gastric mucus membrane with a small strip of squamous epithelium above.

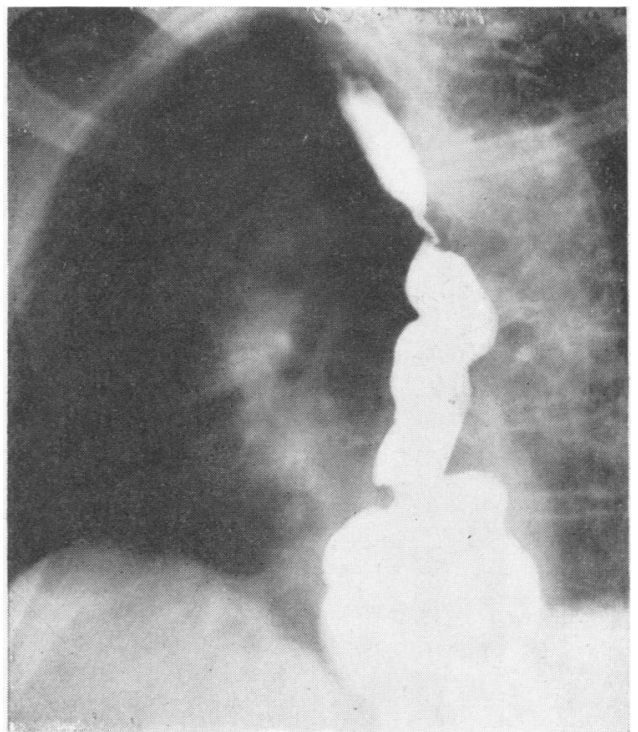

Fig. 3

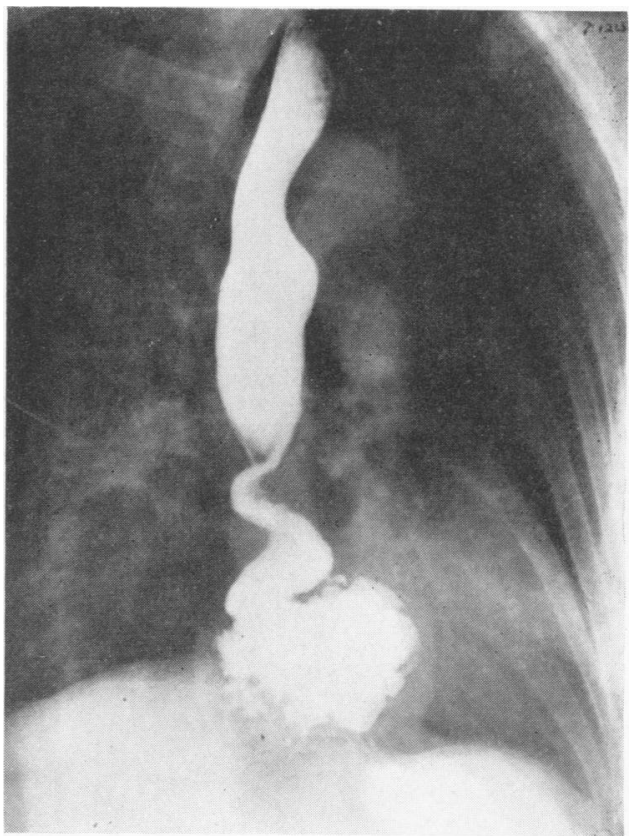

Fig. 4 


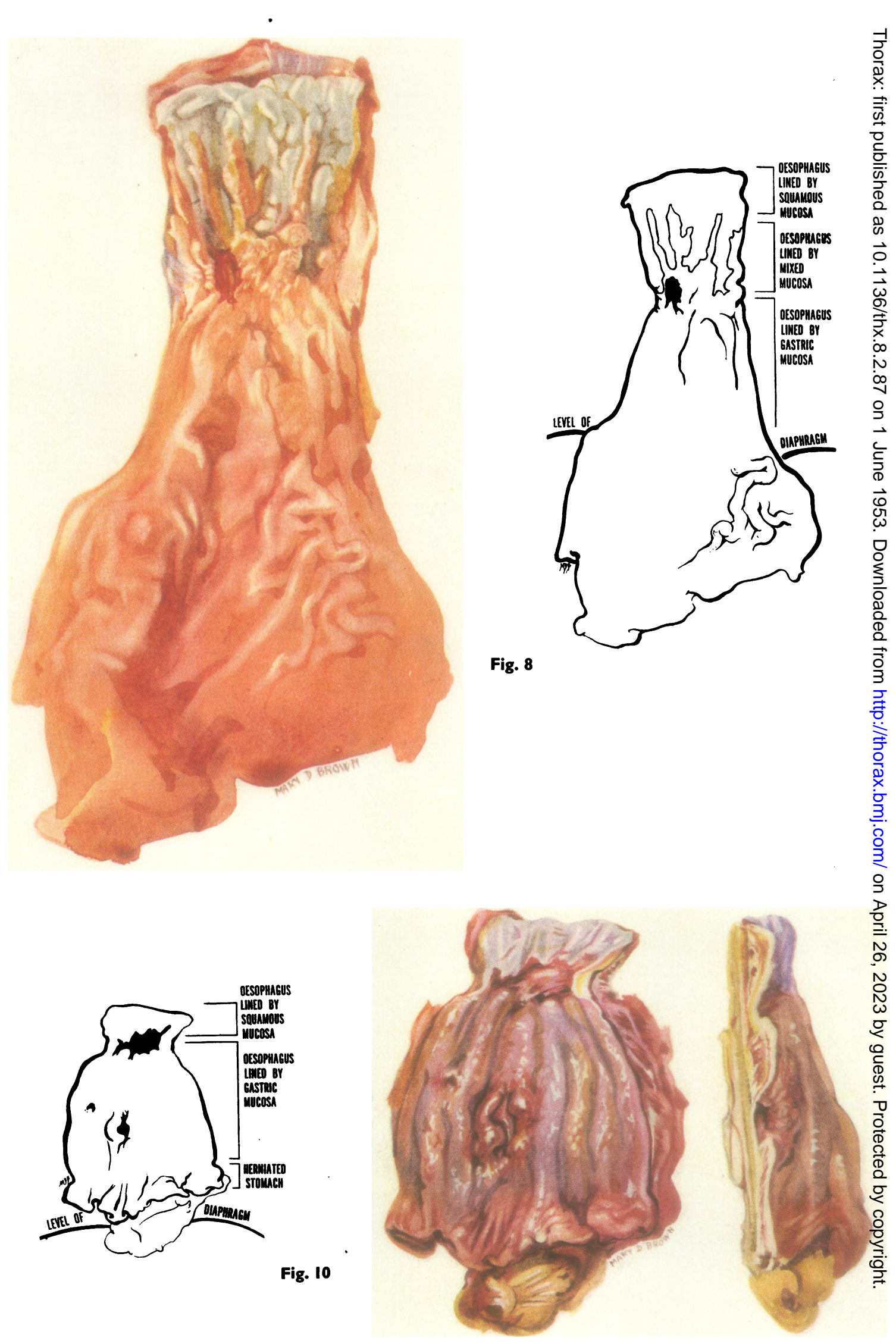




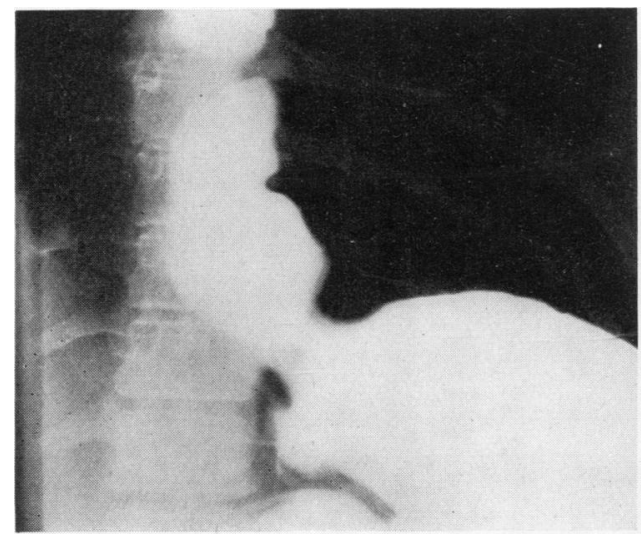

FIG. 6

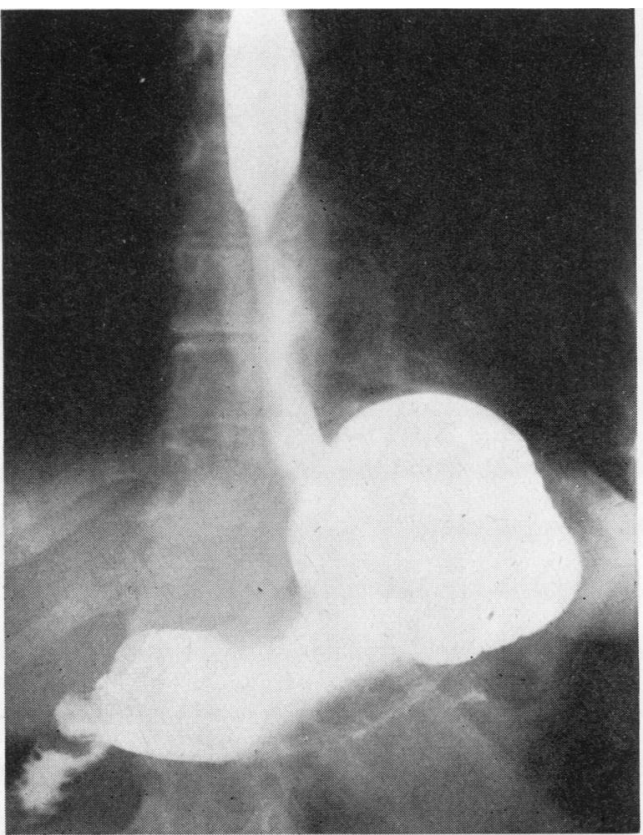

FIG. 7

FIG. 6.-Radiograph of Case 5 before the first operation. This shows the three segments as in Cases 2 and 3.

FIG. 7.-Radiograph of Case 5 after reduction of the sliding hernia. The stenosis and oesophagitis persist. The segment from the stenosis to the diaphragm is oesophagus lined by gastric mucous membrane, and reflux from the stomach persists.

Fig. 8.-Excised specimen from Case 5. The junction of squamous and gastric mucous membranes is very irregular. The ulcer is marginal, the upper border being squamous and the lower gastric epithelium.

Fig. 9.-Radiograph of Case 6 showing characteristic three segments.

FIG. 10.-Excised specimen from Case 6 showing a reflux ulcer of the oesophagus with stenosis, Barrett's ulcers of the gastric mucous membrane lining the oesophagus, and a small strip of normal gastric mucosa from the herniated stomach.

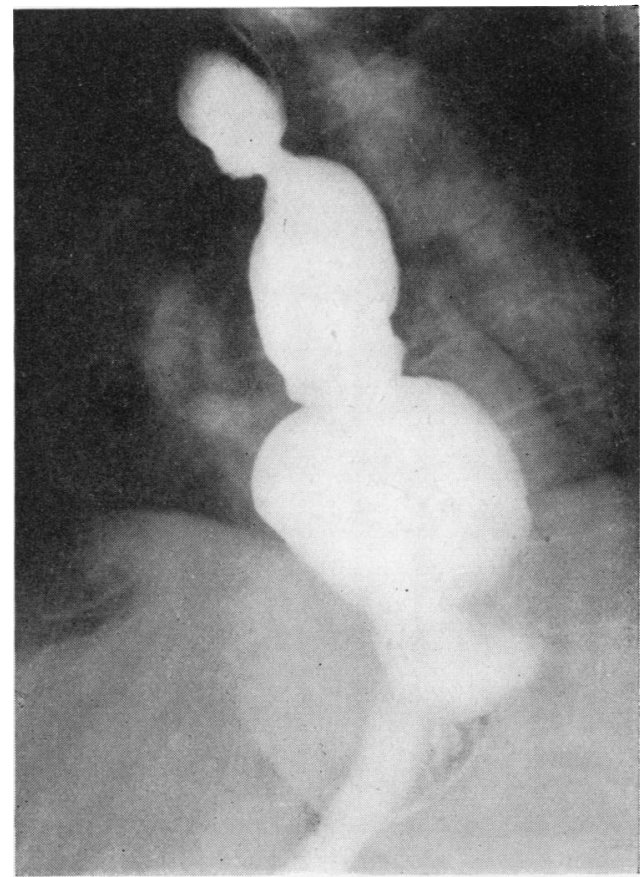

FIG. 9

which from the outside looks like oesophagus and from the inside looks like stomach as "oesophagus lined with gastric mucous membrane." As it is a relatively uncommon aberration, the length of the title will be no great burden.

Bosher and Taylor (1951) described a patient with a stricture of the oesophagus, due to ulceration at the level of the aortic arch, in whom the radiograph showed a small gastric hernia into the mediastinum. When this lesion was resected it was found that the ulcer was in the squamous epithelium of the oesophagus, but that the tube connecting this area with the stomach had the appearance, from the outside, of a normal oesophagus, but was lined with gastric mucous membrane, without oxyntic cells. Oesophageal mucous glands were present in the submucosa. There was no ulceration of the gastric mucous membrane. This was, therefore, an example of reflux oesophagitis with peptic ulceration and stenosis of the oesophagus in a patient whose lower oesophagus was lined with gastric mucous membrane. There is room for argument as to whether the reflux into the squamouslined oesophagus came from the lower oesophagus or from the stomach. It is significant that a small hernia of the stomach was demonstrated radiographically, that the patient was 63 years old, and that all the elderly patients in the present series 


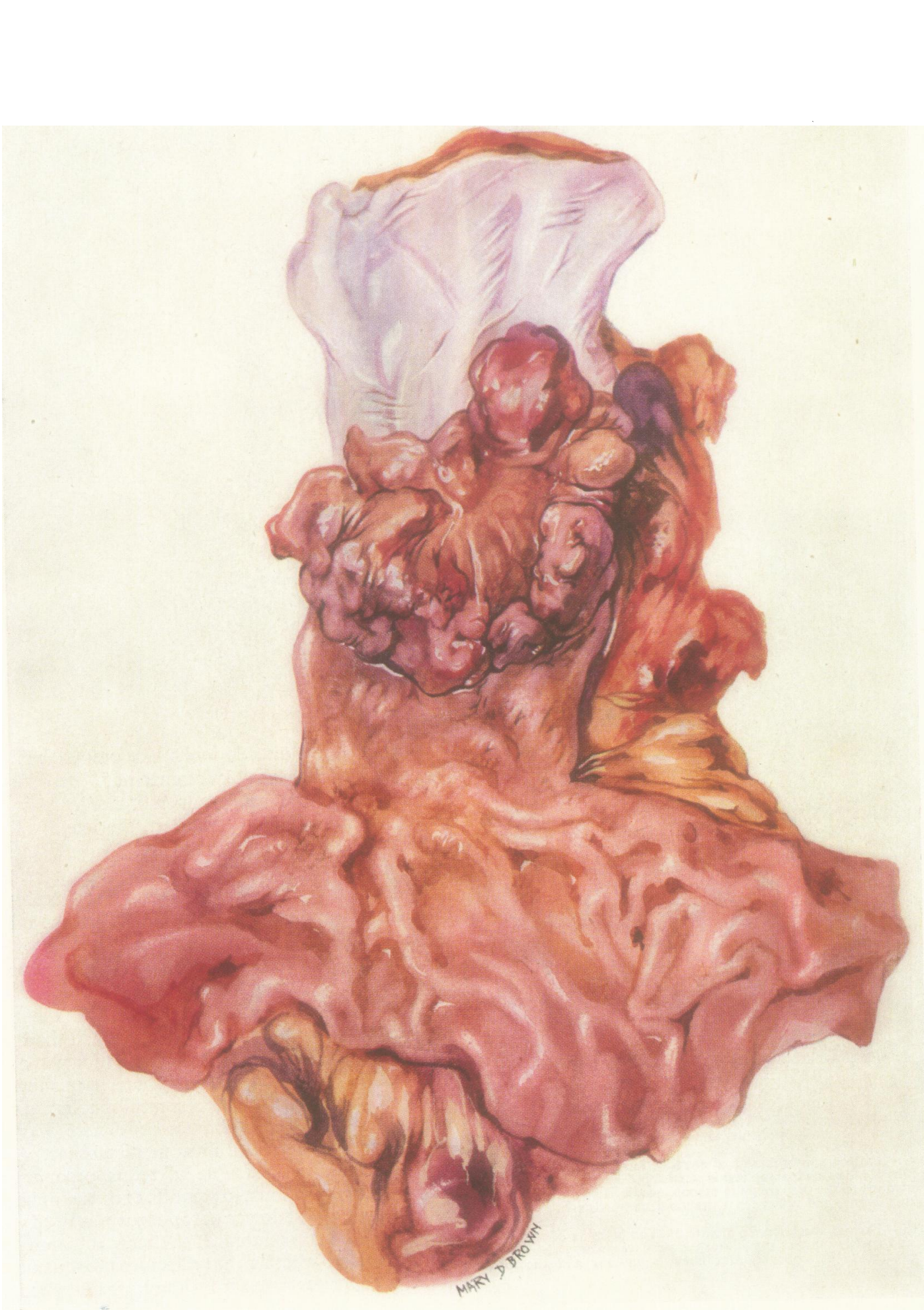

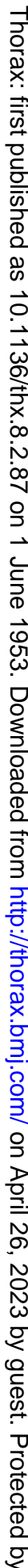

Fig. 12

을 
also had sliding herniae of the stomach. As the lower oesophagus had presumably been lined with gastric mucosa during all the patient's life, there would be some support for a suggestion that the small hernia of true stomach, acquired later in life, was the main cause of the oesophageal reflux. These are, however, conjectures, and the exact role played by the different elements is by no means clear.

The literature, therefore, contains many clinical reports of reflux oesophagitis and pathological reports of Barrett's ulcer. Bosher and Taylor's patient enlarges the picture in that it presents a clinical and pathological description where the stage was set for the development of Barrett's ulcer, but where, in fact, simple reflux oesophagitis with stenosis occurred. The present paper is intended to put a frame round this picture by a clinical, radiological, and pathological description of patients with oesophagus lined with gastric mucous membrane in whom many of the known complications occurred. An effort will be made to put them into some perspective along with the descriptions of the commoner sliding herniae with reflux oesophagitis.

\section{CASE RePORTS}

CASE 1.-M. E. M., a woman aged 66 years, for three years had had recurrent attacks of violent pain in the

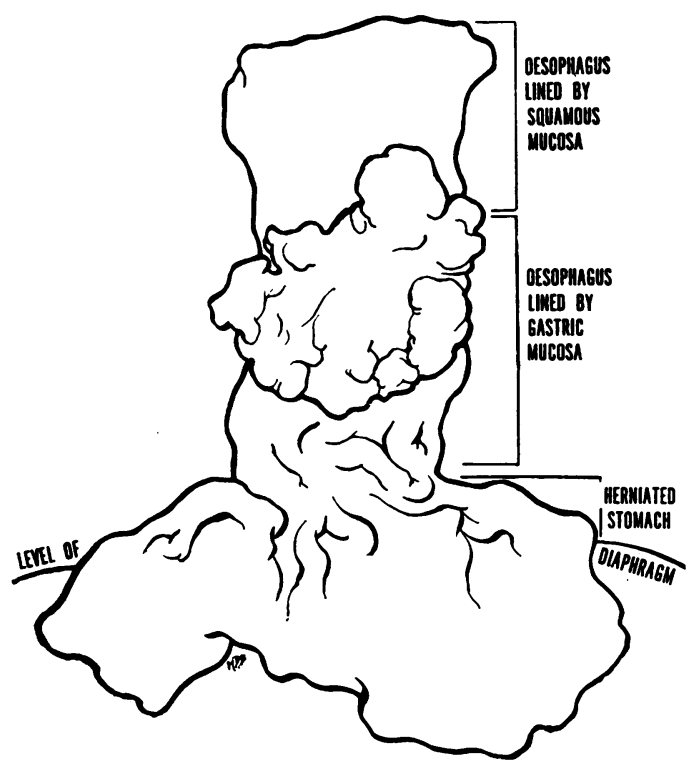

Fig. 12.-Gastric carcinoma of the oesophagus from Case 7 associated with, but not arising from, a sliding hernia of the stomach. The affected area of oesophagus is lined by gastric mucous membrane.

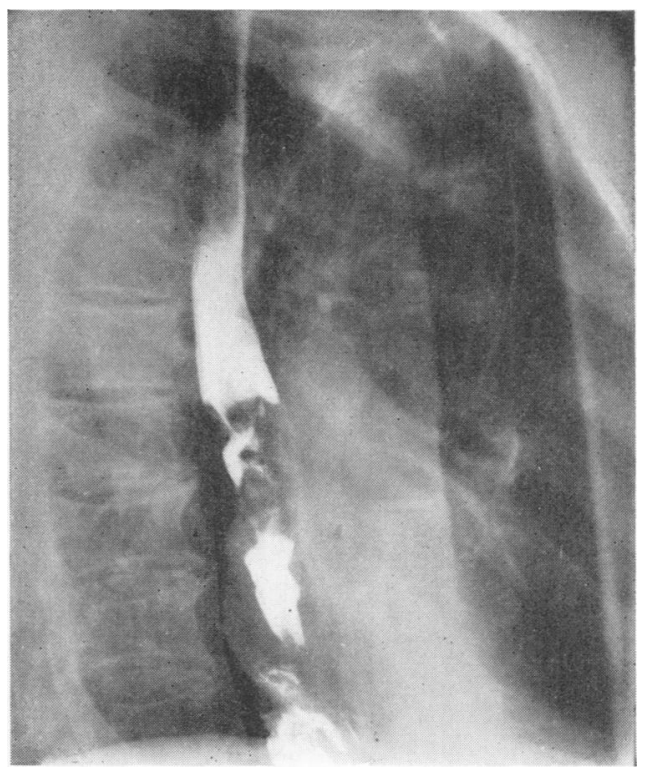

FIG. 11.-Radiograph of Case 7 showing a small sliding hernia of the stomach at the lower end, with an irregular filling defect in the oesophagus above this caused by a carcinoma arising in its gastric epithelial lining.

epigastrium and lower chest behind the sternum. The pain was related to meals, and was relieved by passing the finger into the throat to cause vomiting. The food came back unchanged and not mixed with either acid or bile. There had been a feeling of distension but no belching, no heartburn, and no postural regurgitation. The pain did not occur if the patient limited her meals to coffee and biscuits, and it never came on at night. She had had five children and one miscarriage, without heartburn. She had lost 2 st. in weight in three years. There were no abnormal physical signs. The patient was flabby, weighed 10 st. 2 lb., and was very miserable.

Radiographic examination was performed with the following, report :

" The heart was displaced a little to the left and there was some unfolding of the aorta. Behind the heart a hernia of the stomach was seen. No abnormality was detected in the upper and middle parts of the oesophagus. Immediately above the diaphragm there was a small dilatation or diverticulum projecting downward and to the right. The cardia lay above the diaphragm. A fairly large pouch of stomach above the diaphragm was filled, and the barium passed from this through the hiatus into the lower compartment. In the upper pouch the mucosa appeared to converge towards the posterior wall as if a crater were present, but none could be detected with certainty. With the patient erect there was some return of the stomach to the abdomen, but the fundus appeared to be firmly anchored within the sac. The rest of the stomach was normal. A moderately large duodenal diverticulum was found and another at the beginning 
of the jejunum. The duodenal cap was normal. No reflux into the oesophagus was observed."

It seemed from this that the patient had a mixed type of hiatal hernia, partly sliding and partly paraoesophageal. The violent pain and the radiograph suggested the possibility of a gastric ulcer in the para-oesophageal herniated pouch of stomach. It caused surprise, therefore, when at oesophagoscopy some superficial oesophagitis with leukoplakia was found at $23 \mathrm{~cm}$. from the incisor teeth, and gastric mucosa encountered at $24 \mathrm{~cm}$. There was no stenosis at the junction. As the oesophagoscope traversed the gastric mucosa, some bleeding occurred. Although no ulcer was seen, a note was made that it would have been easy to miss one among the exuberant folds of mucosa.

A diagnosis was then made of mild oesophagitis with leukoplakia, lower segment of oesophagus lined by gastric mucosa, sliding and para-oesophageal hiatal hernia, and possible gastric ulcer.

Operation.-Through a left thoraco-abdominal incision the viscera were explored. A large sliding hernia of the stomach with an added para-oesophageal pouch was found. The oesophagus was about $10 \mathrm{~cm}$. long below the aortic arch, and it entered the top of the hernia about $10 \mathrm{~cm}$. above the diaphragm. The vagus nerves were taut along the surface of the oesophagus and spread out on to the stomach, and there was no doubt from this and from the way the arteries entered the oesophagus directly from the aorta that this was a congenitally maldeveloped oesophagus. No ulcer was palpable. A fibrotic gall-bladder full of stones was present. Resection of the oesophagus was performed from above the aortic arch, and the herniated part of the stomach was removed with it. The remaining portion of stomach was closed and continuity restored in the superior mediastinum by oesophago-jejunostomy by Roux loop. The gall-bladder was removed. The patient made a steady recovery.

The report on the specimen (Fig. 1) by Dr. D. H. Collins was as follows :

Naked Eye Examination.- “The specimen comprises a substantial portion of the body and cardia of stomach, measuring about $13 \mathrm{~cm}$. horizontally and $7 \mathrm{~cm}$. vertically, together with $5 \mathrm{~cm}$. of oesophagus, the mucosa of which, at the upper end, has shrunk down from the muscle during fixation. There is no sharp demarcation of mucosa at the anatomical cardia, although the mucous lining appears to become ironed out in the oesophagus.

" The oesophagus was separated with a knife from the stomach along the line of peritoneal reflection. A vertical slice was then made through the centre of the reconstituted specimen, i.e., up the posterior wall, and three vertically contiguous blocks were prepared, and a horizontal block then made across the extreme upper limit of the oesophageal mucosa."

Histology.-CThe stomach below the anatomical junction with the oesophagus is lined by gastric mucosa of fundal type. The submucosa is oedematous and congested. Cardiac glands and cardiac gastric mucosa do not appear until $0.6 \mathrm{~cm}$. up the anatomical oesophagus, and oesophageal glands appear at $2 \mathrm{~cm}$. up the oesophagus underlying columnar-cell mucous membrane. The first islet of squamous epithelium appears $3 \mathrm{~cm}$. up the anatomical oesophagus, but predominantly gastric mucosa continues to the upper limit of the specimen. The transverse sections across the uppermost part of the removed mucosa show alternations of squamous and gastric mucosa.

"The rather villous type of cardiac mucosa, its lack of depth, and a diffuse fibrosis of the submucosa in the zone for 2 to $4 \mathrm{~cm}$. above the stomach orifice, suggest healing of previous shallow ulcerations."

Comment.-The clinical diagnosis of reflux oesophagitis, lower oesophagus lined with gastric mucosa, and mixed sliding and para-oesophageal hernia was, therefore, confirmed. It seems likely from the histological report that there had also been recurrent superficial "gastric ulcers of the oesophagus," but that none of these had become chronic.

CASE 2.-D. F., a woman aged 56 years, started to have severe cramp-like pains behind the sternum seven years before she attended hospital. The pain was brought on by swallowing. For two years she had frequently had regurgitation of food and saliva and she would often awake choking in the night to find food and mucus in her throat.

Radiographic examination (Fig. 2) was reported as follows :

"A persistent narrowing of the oesophagus was noted at the level of the aortic arch. A mixed sliding and para-oesophageal hiatal hernia was present with a large pouch and very free regurgitation into the oesophagus. The length of oesophagus from the aortic arch to the cardia showed some irregular secondary waves and was a little dilated and hypotonic. This may be due to constant reflux."

Oesophagoscopy showed oesophagitis with superficial ulceration at $28 \mathrm{~cm}$. from the incisor teeth. There was some narrowing of the oesophagus by the inflammation at this level, but the instrument could be passed through it. Immediately below the stricture, gastric mucosa was seen. A pinch biopsy at $31 \mathrm{~cm}$. showed gastric mucosa with goblet cells and occasional oxyntic cells, and one at $41 \mathrm{~cm}$. was reported as being gastric mucosa with oxyntic cells.

The patient was so much improved after oesophagoscopy that she decided against operation, and no gross specimen is available.

The lesion is clearly one of peptic ulceration of the oesophagus with stenosis, a lower segment of oesophagus lined with gastric mucosa, a sliding hernia with a large para-oesophageal sac.

CASE 3.-A. T., a woman aged 78 years, had had good health until two years before attending hospital. For these two years she had complained of pain in the lower sternal region going through to the back after 
meals. This was associated with heartburn when she lay down. For about six months she had noticed food sticking at the level of the manubrial notch and the regurgitation of saliva. She was a rather thin old woman with marked arteriosclerosis.

Radiographic examination (Fig. 3) was reported as follows:

"Some enlargement of the heart with dilatation and unfolding of the aorta was seen. There was a persistent fine narrowing of the oesophagus at the level of the aortic arch. Below this was a relaxed segment of oesophagus ending in a large sliding hernia of the stomach. Reflux was free from the gastric pouch into the oesophagus as far as the aortic arch, when further reflux was prevented by the stricture."

Oesophagoscopic examination showed fluid retention and intense oesophagitis down to $21 \mathrm{~cm}$., at which level there was chronic ulceration with a tight inflammatory stenosis. The stricture dilated easily so that the instrument could be passed through it. Immediately below the stenosis gastric mucous membrane was found, and it was thought that a change in the character of this could be detected at $29 \mathrm{~cm}$. Biopsies were taken and reported on as follows:

"At $21 \mathrm{~cm}$. was a portion of mucosa covered with stratified squamous epithelium in which there was simple ulceration; at $26 \mathrm{~cm}$. fragments of mucosa, covered with mucus-secreting glandular epithelium, there being numerous mucous glands in the subepithelial tissues and heavy lymphocytic, plasma cell, and eosinophilic infiltration; and at $33 \mathrm{~cm}$. a portion of gastric type mucosa."

This was another example of reflux peptic ulceration of the oesophagus with stenosis occurring in a patient with a sliding hernia of the stomach and a lower segment of oesophagus lined with gastric mucous membrane. In view of the patient's age and arteriosclerosis, operation was not advised.

CASE 4.-J. E. H., a woman, aged 65 years, had had a windy dyspepsia for many years, with intermittent but increasing dysphagia for seven years. Food seemed to lodge behind the lower half of the sternum, causing a sense of constriction which was relieved by self-induced vomiting. For two months before attendance at hospital she was able to swallow only fluids and had sometimes had difficulty with these. The patient was very weak, thin, and kyphotic.

Radiographic examination (Fig. 4) was reported as follows :

"There is a stricture of the oesophagus at the level of the eighth dorsal vertebra with some dilatation above. The stricture is about $2 \mathrm{~cm}$. long, is irregular, and shows a small ulcer crater along its course. Distal to the stricture, and above the hiatus, is a large pouch lined by gastric mucosa. This is not reducible, and regurgitation is free."

Oesophagoscopy showed moderate inflammation with oedema of the squamous epithelium at $27 \mathrm{~cm}$. A tight stricture was found at $30 \mathrm{~cm}$. On the right anterolateral wall, at the level of the stricture, the mucosa was thrown into coarse folds as though it were gastric. A bougie passed into the stricture was caught up in folds of gastric mucosa below, and dilatation was not complete. The appearances were not quite those of peptic ulcer of the oesophagus and the possibility of the lesions being a gastric ulcer in a herniated pouch was considered. A biopsy from the region of the stenosis showed cellular fibrous and granulation tissue. A second small biopsy showed hyperplastic squamous epithelium and a small strip of gastric mucosa of cardiac type. Isolated clumps of polymorphonuclear leucocytes were also present.

Four days after examination the patient suddenly felt a severe stabbing pain under the left breast which made her very distressed. She could not lie down, and was admitted to hospital the following day short of breath but not cyanosed. The pain was felt right through the chest to the back, and was not affected by coughing. A tentative diagnosis of subacute perforation of the ulcer with local mediastinitis was suggested.

A left thoraco-abdominal approach was made. The posterior mediastinal pleura was thickened, scarred and puckered, and at a point $5 \mathrm{~cm}$. below the aortic arch the appearances were reminiscent of the peritoneal changes over a chronic gastric ulcer. There was much thickening and inflammation of the neighbouring mediastinal tissue. A sliding hernia of the stomach was present, and the junction of oesophagus and stomach was about $5 \mathrm{~cm}$. above the hiatus. The oesophageal stenosis was $2 \mathrm{~cm}$. above the apparent cardia. It had to be cut away from the right lung and pleura with a knife. The lower oesophagus and upper part of the stomach were resected, and continuity restored by a Roux loop anastomosed to the oesophagus below the aortic arch. The patient recovered normally.

When the specimen was opened a narrow strip of stratified epithelium was seen at the top. Below this the oesophagus was lined with gastric folds and contained a perforating, stenosing ulcer (Fig. 5). The lower part was true herniated stomach.

Dr. T. W. Sutherland reported as follows on the fixed specimen:

Pathology._."A piece of stomach and attached oesophagus measures $7.5 \mathrm{~cm}$. in length and $9 \mathrm{~cm}$. in its widest part. A narrow part, $3 \mathrm{~cm}$. broad and $5 \mathrm{~cm}$. long, is apparently oesophagus mainly lined by gastric mucosa. In the middle of this is an ulcer measuring $5 \mathrm{~mm}$. transversely and $7 \mathrm{~mm}$. longitudinally. There is complete loss of the muscularis with peri-oesophageal fibrosis in relation to the ulcer. Histological examination shows the upper end of the oesophagus for $5 \mathrm{~mm}$. lined by stratified squamous epithelium. The rest is lined by gastric mucosa of which the proximal two-thirds is of atrophic cardiac type without oxyntic cells. In the centre of this lies the ulcer. The lower third of the specimen is lined by fundal type of gastric mucosa with oxyntic cells. The ulcer itself is covered with purulent exudate resting on a necrotic zone overlying granulation tissue infiltrated with leucocytes. Deep to the ulcer, muscle is replaced by fibrous tissue." 
This patient had a sliding hiatal hernia and a lower oesophagus lined with gastric mucosa which was the seat of a perforating and stenosing gastric ulcer.

CASE 5.-A. B. H., a woman aged 48 years, had suffered from intermittent pain after meals, flatulence, and heartburn for six years, and for two years had had dysphagia. Food seemed to meet an obstruction "like a marble" about the middle of the sternum. She would regurgitate mucus, but never brought her food back. She had had much discomfort and vomiting with her two pregnancies, but these symptoms had passed off when the children were born. There were no abnormal physical signs and the patient's condition was good.

Radiographic examination (Fig. 6) elsewhere was reported as follows:

"There is a sliding hernia of the fundus of the stomach up to about $8 \mathrm{~cm}$. above the hiatus of the diaphragm. At the upper limit of this is a narrow segment of oesophagus $7 \mathrm{~mm}$. across and $1 \mathrm{~cm}$. long which is almost certainly a stenosing peptic ulcer of the oesophagus."

Oesophagoscopy showed the oesophagus to be filled with green bilious fluid. There was intense superficial oesophagitis from the level of the crico.d cartilage downwards. At $30 \mathrm{~cm}$. there was an inflammatory stenosis $\frac{1}{2}$ to $1 \mathrm{~cm}$. long, which was soft and dilated easily. A biopsy was taken from the stricture, and the oesophagoscope passed on into a gastric pouch beyond. The biopsy showed an ulcer, in the floor of which fibrinoid necrosis and polymorph exudate were present. The tissue was partly covered with gastric mucosa of cardiac type.

At this time it was wrongly assumed that the ulcer was a stenosing peptic ulcer of the oesophagus caused by reflux. It was also thought that it might be possible for a fairly acute inflammatory process in the submucosa to cause a stenosis, and that this might resolve if the causal reflux were stopped by adequate reduction of the hernia. It has since been learnt that neither of these hypotheses is tenable. However, at that time an operation was performed to reduce the hernia of the stomach. The cardia was found to be $5 \mathrm{~cm}$. above the hiatus. Immediately above the cardia, and closely applied to the wall of the oesophagus, was a cluster of inflamed lymph nodes, and above this the wall of the oesophagus was rather firm and attached by fibrous tissue to the pericardium. The oesophagus was mobilized, the vagi remaining intact, and the hernia was reduced and fixed below the diaphragm. After this operation the patient's symptoms persisted, and a further radiograph (Fig. 7) showed the stenosis half-way between the aortic arch and the diaphragm, with herniated stomach apparently still present below it. Oesophagoscopy this time showed severe oesophagitis with ulceration and stenosis at $26 \mathrm{~cm}$. and gastric mucosa below this. A second operation was therefore performed. When the chest was opened the stomach was not to be seen in the mediastinum. The oesophagus extended through the hiatus, and the fundus of the stomach was well anchored below the diaphragm. The oesophagus below the aorta, and the upper end of

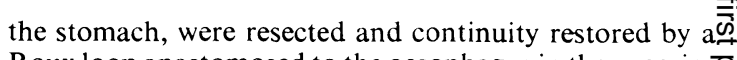
Roux loop anastomosed to the oesophagus in the superior? mediastinum. Examination of the specimen (Fig. 8)ㅡㅡ showed that nearly the whole of the excised oesophaguse was lined with gastric mucosa, and at its upper end thereo were digitations of squamous epithelium as much as $2 \mathrm{~cm}$. long. An ulcer was also present, and Dr. D. H. Collins reported as follows:

Naked Eye Examination.-." The specimen comprises $\overrightarrow{ }$ the lower $6 \mathrm{~cm}$. of oesophagus and a part of the stomach ${ }_{\sigma}^{\omega}$ laid open along the line of the lesser curvature and the right border of the oesophagus. On the outer surfaces $x$ the anatomical junction of oesophagus with stomach is apparent, and is outlined by the reflection of the $e_{\infty}^{N}$ peritoneum, but the junction cannot be distinguished on the mucous surfaces, since columns of infolded gastric을 mucosa ascend the oesophagus, blending with the squamous epithelium in a very irregular way. A smalle sharply defined ulcer, $10 \quad 7 \mathrm{~mm}$., lies on the posterior wall of the oesophagus, $3.5 \mathrm{~cm}$. above the anatomical gastro-oesophageal junction."

Histology._. " A consecutive series of sections of ${ }^{\text {c }}$ stomach and oesophagus were prepared from a vertical slice of the tissue midway between the posterior and left lateral oesophageal walls. This slice included $3 \mathrm{~cm}$. of stomach wall in the same plane. The stomach, up to its anatomical junction with the oesophagus, is lined by normal gastric mucosa with glands of fundal type. Cardiac glands only are seen in the immediate neigh-을 bourhood of the gastro-oesophageal junction, and 3 cardiac type of gastric mucosa extends up the oesophagus for $3.0 \mathrm{~cm}$. before joining with continuous stratified? squamous epithelium. Small islands of squamous epithelium, however, interrupt the gastric mucosa at음 two points, $1.7 \mathrm{~cm}$. and $2.5 \mathrm{~cm}$. above the gastrooesophageal junction, and, corresponding with the first appearance of squamous epithelial islands, oesophageal 3 . glands and ducts appear in the submucosa. A diffuse inflammatory cellular reaction, comprising mainly lymphocytes and plasma cells, but including some eosinophil and neutrophil polymorphs, is notable in the 9 mucosa and submucosa for $1 \mathrm{~cm}$. above the squamous- $\rightarrow$ columnar epithelial junction, and two shallow erosions

of squamous epithelium are seen here. the ulcer of the oesophagus noted above. This is an ulcer extending across the junction between gastric and $\mathbb{W}^{2}$ squamous epithelium. In its upper part islands of 0 squamous epithelium remain, and oesophageal mucous glands and ducts are prominent in the ulcer floor. Gastric mucosa of cardiac type forms the lower border of the ulcer, and one island of columnar-cell epithelium lies about the middle of the ulcer. The ulcer is of some 뭉 chronicity with a cicatrized floor, but the muscular wa!l is little affected."

This was an example of severe reflux oesophagitis $\frac{\stackrel{?}{\mathbb{Q}}}{\mathbb{Q}}$ with ulceration, a lower oesophagus lined by gastric mucosa, and a sliding hernia of the stomach which had been reduced at a previous operation. Although superficial ulceration was present in the 
squamous-lined unresected part of the oesophagus, the chronic ulcer causing stenosis was marginal, being surrounded partly with squamous and partly with gastric epithelium.

CASE 6.-M. J. S., a woman, aged 59 years, for three years had complained of heartburn and a bitter taste in the mouth on stooping or when lying down in bed. Occasionally vomiting would relieve her discomfort. She had also had much flatulence. For six months food seemed to stick behind the middle of the sternum, causing choking and regurgitation. Before she attended the hospital she reached the stage when she could only swallow fluids. She was a very fat, pale woman with poor posture and poor chest expansion, but there were no abnormal physical signs.

Radiographic examination (Fig. 9) was reported as follows:

"Barium passed down the oesophagus normally into a herniated pouch of stomach. There was slight rigidity and constriction at the level of the aortic arch suggesting some ulceration, but this is some way above the top of the obvious gastric hernia. The oesophagus, between the constriction and the hernia, is abnormal and probably affected by oesophagitis. Reflux of barium is free. There is a suggestion of more than one ulcer crater."

At oesophagoscopy moderate superficial oesophagitis was noted from 20 to $25 \mathrm{~cm}$. At $25 \mathrm{~cm}$. there was deep ulceration with constriction, but this could be gently dilated to allow the oesophagoscope to pass. The mucosa immediately beyond the constriction was pink and oedematous and thrown into typical gastric folds. A biopsy proved it to be gastric epithelium.

It was thought that the patient was not a suitable subject for an extensive resection, and so she was sent home on a soft and fluid diet, with instructions to return for dilatation of the stricture as necessary. While at home, however, she had a very severe haematemesis and had to be readmitted to hospital.

A left thoraco-abdominal incision was made through the bed of the eighth rib. A sliding hernia of the stomach into the mediastinum was found. Into the top of this a perfectly normal looking oesophagus entered as in any other sliding hernia. The junction of oesophagus and stomach was about $6 \mathrm{~cm}$. above the hiatus. There was no external evidence of oesophageal constriction at any level, and if it had not been for the knowledge gained at oesophagoscopy the hernia would have been reduced in the usual way. The fact that stenosis was present at the aortic arch, and that gastric mucosa was present in the oesophagus below this, necessitated an extensive resection. The oesophagus and herniated stomach were therefore removed, the pyloric end of the stomach closed, and oesophago-jejunostomy performed in the superior mediastinum by a Roux loop extending through the hiatus, along the mediastinum deep to the aortic arch. Although the patient herself was so fat, the mesentery of the small intestine was thin and pliable. The patient recovered without incident.

The following report on the specimen (Fig. 10) was received from Dr. T. W. Sutherland:
Pathology._-"The oesophagus consists of an upper segment $1.5 \mathrm{~cm}$. long lined by squamous epithelium separated by an ulcerated constricted area from a lower segment about $7 \mathrm{~cm}$. long, which is lined by coarse, pale pink, longitudinal folds of gastric mucous membrane. Slightly below the middle of this area is a deep penetrating gastric ulcer, and above and to one side is a smaller but similar one. The lower end of the specimen consists of a portion of stomach, and here the mucosa is less swollen and a deeper pink than that lining the lower segment of oesophagus. Histological examination shows that the upper ulcer has involved that part of the specimen covered by stratified squamous epithelium. The distal border of the ulcer is formed by a short cuff of stratified squamous epithelium; distal to this cuff the lining is gastric. The floor of the upper ulcer is covered by a purulent layer overlying a zone of fibrinoid necrosis, with enmeshed degenerate pus cells; the latter merges into a deeper granulation tissue layer, lightly infiltrated with degenerate polymorphs, occasional eosinophils, and more densely arranged lymphocytes. There is no fibrous replacement of muscle. Between the distal margin of the upper ulcer and the proximal margin of the lower the specimen is covered by mucosa of cardiac type; the glands are simple tubules rich in goblet cells. Oxyntic cells are absent. Oesophageal mucous glands lie in the submucosa. The distal ulcer displays a floor covered by purulent exudate, with underlying granulation tissue lightly and diffusely infiltrated with degenerate polymorphs, lymphocytes, and eosinophils. A fibrinoid layer is lacking. Ducts of underlying oesophageal mucous glands are dilated. The submucosa is fibrotic, but there is no fibrous replacement of muscle. Distal to the lower ulcer the tissue is covered by gastric mucosa of fundal type, rich in oxyntic cells. It is possible that the atrophic mucosa between the two ulcers may cover an area, previously ulcerated, which has now healed."

The patient combined in one specimen an oesophagus lined with gastric mucous membrane, a sliding hernia of the stomach, reflux stenosing oesophagitis, and two Barrett's ulcers, one of which had recently caused severe bleeding.

CASE 7.-L. W. B., a man aged 72 years, had had hunger pains on and off all his life, with flatulence and occasional nocturnal regurgitation of a fluid that made him choke. At the age of 52 he had severe epigastric pain, and a similar attack, with pain shooting under the left costal margin, 18 years later. There had been several attacks of severe and prolonged hiccup. For eight weeks he had increasing dysphagia, the food seeming to stick at the level of the manubrium sterni. There was no abnormality to be detected on clinical examination.

The radiological report (Fig. 11) was as follows:

"There is a filling defect in the lower half of the oesophagus, $5 \mathrm{~cm}$. long and starting $5 \mathrm{~cm}$. below the bifurcation of the trachea. - There appeared to be an ulcer crater in the middle of the filling defect. Below there was a short length of normal-looking oesophagus, which ended in a small sliding hernia of stomach. The 
rest of the stomach appeared normal, but gall-stones were present."

At oesophagoscopy the oesophagus looked normal down to $30 \mathrm{~cm}$., at which level there was a soft, proliferating mass causing partial obstruction. A biopsy was reported as adenocarcinoma. Bronchoscopy showed no distortion of the bronchial tree.

Operation.-At operation the presence of a sliding hernia was confirmed, the cardia being $5 \mathrm{~cm}$. above the hiatus. Above this the oesophagus looked quite normal. There was no evidence of mediastinal thickening or any metastases. Only when the oesophagus was isolated could soft tumour within it be felt extending nearly to the arch of the aorta. The oesophagus was resected from $5 \mathrm{~cm}$. above the top of the tumour, the fundus of the stomach and left gastric vessels and cellular tissue being taken with it, and intestinal continuity restored by a Roux loop anastomosed to the oesophagus in the superior mediastinum.

Dr. D. H. Collins reported on the specimen (Fig. 12) as follows:

Naked Eye Examination.- " The specimen comprises a part of the cardiac portion and body of the stomach together with $9 \mathrm{~cm}$. of oesophagus. The lower oesophagus is occupied by a large fungating carcinoma, arising $1 \mathrm{~cm}$. above the line of cardiac orifice, and extending upwards for 4.5 to $6 \mathrm{~cm}$. before giving way to pale squamous epithelium. The growth, anatomically, is entirely lying within the oesophagus, and arising from oesophagus. The left gastric vessels and a mass of omentum are also attached. This may contain some small tumour-infiltrated glands."

Histology._- "The tumour is an adenocarcinoma of gastric-type epithelium. It is largely polypoidal, but also penetrates through the muscular coats of the oesophagus to reach the adventitial connective tissues, where invasion of lymphatic channels by the tumour is also seen.

"The epithelium above the growth is entirely squamous; below the growth it is gastric in type, and shows cardiac glands and a remarkable submucous infiltration by large eosinophilic cells resembling plasma cells swollen by Russell bodies.

"The tumour, on the whole, maintains a fair degree of differentiation."

This case presented the same anatomical abnormality of sliding hernia and oesophagus lined with gastric mucous membrane seen in the other patients, but the dysphagia which brought the patient to hospital was caused by an adenocarcinoma in the gastric lining of the oesophagus.

\section{DiSCUSSION}

Since Barrett (1950) published his account of gastric ulcers of the oesophagus a review of all patients found to be suffering from peptic stenosis of the oesophagus attending the Thoracic Surgical Department of the General Infirmary at Leeds has been made, the symptoms and oesophagoscopic and radiological reports being carefully checked. It would have been more significant if this could have been done with the many hundreds of diaphragmatic herniae that have been studied, but it is obvious that shallow ulcers in gastric folds could so easily be missed at oesophagoscopy that the findings, viewed in retrospect, would too often be inconclusive for the investigation to be profitable at this time. The peptic stenoses, however, have been more easy to assess and, as all the radiographs were available for examination, it was not necessary to rely on reports alone. From this study it appears that, while there have been 115 patients with stenosis from oesophageal ulcer, there have bsen 10 with stenosis from Barrett's ulcer, and 23 in whom the evidence still left doubt about the exact morbid anatomy. Of the 115 patients with oesophageal ulcer and stenosis, there was indisputable evidence of a segment of oesophagus lined with gastric mucosa between the stenosis and the hernia in 11. These figures must not be taken to indicate either the frequency of gastric mucosa in the oesophagus, which was 21 out of 125, or of Barrett's ulcer, which was 10 out of 125 , but they do give a very rough idea of the relative chances of a peptic stenosis being oesophageal or gastric, about 10 to 1 .

The radiological investigation of the lower end of the oesophagus needs some revision in the light of increasing experience and opportunities to examine pathological specimens. It is recognized that a pouch of stomach above the diaphragm should fill with barium if the patient lies supine or in a Trendelenburg position. The size of the pouch, the mucosal pattern, and the continuity with the rest of the stomach below the diaphragm are the distinguishing features. In the simple sliding hernia the oesophagus joins the pouch of stomach at its apex, and a slight constriction at the junction represents the cardia. The fine longitudinal folds of oesophageal mucous membrane are sometimes seen to change abruptly to the thickened folds of $\mathrm{N}$ the lining of the stomach, but it has become apparent $N$ that too much stress should not be placed on this for the diagnosis of a hernia, and greater attention has been paid to the physiological action of the cardia.

When it was found that about $75 \%$ of patients $\mathscr{\mathscr { Q }}$ with gastric reflux had macroscopic oesophagitis it was concluded that the determination of the competency of the cardia was more important $\overrightarrow{\mathbb{D}}$ than the demonstration of small pouches, whose $\frac{\vec{D}}{\mathbb{Q}}$ identity might be suspect. Efforts to demonstrate oesophagitis have met with variable success. If the inflammation is superficial there are no charac- 8 teristic changes, but where stenosis occurs it may 
be short and slight, short and tight, or long and rigid. The stricture extends upwards from immediately above the gastric pouch, affects the whole circumference equally, and, where elongated, it may appear truncated. Although ulcers are common, their demonstration is difficult, for they are very superficial and their base is usually covered with slough. Above the stenosis the oesophagus may be dilated according to the amount of obstruction. Peristalsis is usually a normal descending wave, but occasionally in elderly people this is followed by contractions of the circular fibres giving a rippled appearance as the oesophagus empties. In contrast to erosion of squamous epithelium, true gastric ulcers present quite a different radiological picture. When a deep ulcer crater can be clearly demonstrated close to the cardia, there is a strong presumptive evidence of its gastric origin. Such craters may be more than a centimetre in diameter. The walls are clear-cut, and in one case terracing was observed. Undermining of the margins has also been seen, but the edges appeared regular and smooth. The crater is usually single, but in two instances a second crater has been found. They are more often on the posterior or postero-lateral wall, and they tend to spread longitudinally rather than to encircle the lumen. In some the crater lies in a stenotic segment, but in others the lumen is scarcely narrowed and there is little obstruction. Peri-oesophageal infiltration is not uncommon, and soft tissue shadows may be seen at the base of the ulcer.

Examples of hiatus hernia, in which gastric mucosa lines the whole of the lower oesophagus, have provided new features of considerable radiological interest. One of the most striking is the absence of any evidence of change in the mucosal pattern of the oesophagus, unless there are inflammatory complications. As mentioned earlier, it has often been possible, with fair accuracy, to localize the mucosal junction in the terminal oesophagus both under normal conditions and in the presence of a hernia, but when the junction of oesophageal and gastric mucous membrane lies at a higher level, sometimes as high as the aortic arch, the mucosal pattern shows no demonstrable change. It has also been found in these patients that the cardia is lax and reflux very free. The lower oesophagus tends to be hypotonic and is frequently distended with fluid or air. The mucosal folds are thus so flattened that it may not be possible to bring them into radiological relief.

If true reflux oesophagitis with stenosis occurs, then the stricture so formed marks the lower limit of squamous epithelium. The oesophagus below this, which is lined by gastric mucosa, retains its tubular contour, although it may be a little dilated. The position of the cardia can be identified where the lumen widens again to form the sac of herniated stomach. In these circumstances a typical radiological picture is produced of three segments of barium in the chest, the first being in normal oesophagus, separated from the second or intermediate segment by a stricture; the second segment is separated from what is obviously stomach by what is usually identifiable as cardia. When this picture occurs it can be concluded that the intermediate tubular structure is oesophagus lined by gastric mucous membrane. It will be seen that this conclusion rests upon inference, and not upon a demonstrable change in mucosal pattern. It is dependent upon the occurrence of reflux peptic stenosis of the oesophagus.

Some of the gastric ulcers in the intermediate segment have been found to be too shallow to show on the radiograph. In one specimen the mucosa showed changes of gastritis in addition to gastric ulcers, but nothing abnormal could be shown in the mucosal relief. Such examples bring out the limitations of radiology when applied to detailed study of mucous membrane. On the other hand, combined with oesophagoscopy, it is indispensable to the provision of the complete picture before operation.

The importance of a detailed diagnosis is well demonstrated by reference to Case 5 in this series, where a failure to correlate the position of the stenosis seen at oesophagoscopy with the radiological level of the true cardia led to an unsatisfactory operation being performed. The proper treatment of these patients must depend upon an accurate knowledge of the life history of the ulcers to which they are prone, and the factors which contribute to progress or healing of the ulcers. Only by accumulated experience will it be possible to answer such questions as : Will reflux oesophagitis heal after reduction of the hernia, if this produces competence of the cardia, even when gastric mucosa is left lining the oesophagus; and, if this is so, in what proportion of cases may it be expected to be effective?

The Healing of Reflux and Gastric Ulcers OF THE OESOPHAGUS.-Although the knowledge of the natural history of these simple ulcers of the oesophagus is too incomplete for any accurate prognosis to be made, it is certain that superficial reflux ulcers appear, heal, and reappear, whereas stenosis of the oesophagus from chronic reflux ulceration is a permanent deformity with circumferential fibrosis. The latter must therefore be 
treated by excision, or by palliative measures that must be repeated indefinitely. Barrett's ulcer, however, may heal just as a gastric ulcer in the true stomach may heal, so that, if the fibrosis in its floor does not extend completely round the viscus, the obstruction being completed by inflammatory oedema, healing may cure the obstruction. One such ulcer has behaved like this after jejunostomy feeding, leaving a good lumen and a freedom from dysphagia now for six years. It would seem that complete rest, medical treatment, and possibly jejunostomy are worth considering when the diagnosis of Barrett's ulcer is made, and where for one reason or another resection seems unjustifiable.

Consideration of the healing process raises a further problem: If gastric reflux occurs, and causes ulceration of squamous epithelium without producing stenosis, is healing of the ulcer in an acid medium more likely to be by overgrowth of gastric rather than of oesophageal epithelium ? If this were so it might be that some examples of the gastric mucosa in the oesophagus were acquired rather than congenital. Some of the histological reports already quoted would lend support to such a suggestion. This is a subject that clearly calls for further experimental investigation.

\section{CONCLUSIONS}

Sliding hernia of the stomach into the mediastinum with incompetence of the cardia may cause reflux oesophagitis, and by a process of submucous fibrosis this may lead to oesophageal stenosis.

A variable amount of the oesophagus below the aortic arch may be lined by gastric mucosa of cardiac type. This is presumably, but not necessarily in every case, a congenital abnormality, and in the series presented has always been associated with herniation of the true stomach through the diaphragmatic hiatus, with the cardia lying in the mediastinum. It is not known if this association is constant. Radiological investigation alone is not enough to diagnose this abnormality, as the mucosal pattern gives no clue to the change in the histology. It may be thought to be present when there is a marked difference between the level of the mucosal change at oesophagoscopy and the estimated level of the cardia from the radiograph. The mobility of the cardia in sliding hernia makes it unwise to draw such conclusions from only slight differences in these measurements.

Patients with gastric mucosa in the oesophagus are subject to oesophagitis and peptic stenosis of the oesophagus. When this occurs it is found at the lower limit of the squamous epithelium. In the presence of a hernia this complication gives a typical radiological picture of three segments $\overrightarrow{\vec{\rho}}$ separated by the stricture and the cardia.

Patients with the oesophagus lined by gastric 듬 mucous membrane are subject to gastric ulcers $\frac{\bar{m}}{5}$ occurring in that part of the oesophagus lined $\stackrel{\Phi}{\varrho}$ by gastric mucosa, and it is suggested that, if these become chronic, they might be known as Barrett's ulcers. Such ulcers may occur alone or in association with reflux ulcers of the oesophagus. $\overrightarrow{\vec{\omega}}$ If they do not cause stenosis they may be missed both by the radiologist and the ossophagoscopist. $\vec{x}$ They behave like gastric ulcers elsewhere; they may heal, bleed, perforate, or cause stenosis. N Their pathology is different from peptic ulcer of ${ }^{\infty}$ the oesophagus, and, unlike these, they may respond to medical management.

While dysphagia may be caused by either oesophageal or gastric ulcers, severe bleeding is more likely to come from gastric mucous membrane. $\vec{c}$ The association of peptic ulcer of the oesophagus with duodenal ulcer must, however, be remembered when such a patient presents with haematemesis.

Subacute mediastinal perforation or acute pleural perforation of an ulcer suggests that the ulcer is certainly occurring in gastric mucous membrane $\bar{\varnothing}$ whether this be in the oesophagus or the true stomach. Peptic ulcers of the squamous lining of the oesophagus do not perforate.

Where stenosis is present the finding of gastric mucosa above the stricture is suggestive of Barrett's ulcer. The deep interdigitations seen in Fig. 8 음 show that this is not necessarily always reliable.

If the inflammation is too great for accurateo delineation of the different types of mucosa, and if it is not possible to be certain whether an elevatedô pink area is gastric mucosa or granulation tissue, a Barrett's ulcer may be suspected if the ulceration? and swelling appear to be more on one side than $\triangle$ the other. In stenosis from oesophageal ulcer the submucous fibrosis usually seems to be even around the circumference, although-the chronic ulcer may be localized.

\section{SUMMARY}

Records are presented of seven patients in whom the lower oesophagus was lined by gastric mucousc membrane.

All of these patients had reflux oesophagitis, 0 and in four this had progressed to fibrous stenosis. One of the four also had two gastric ulcers in that part of the oesophagus lined by gastric mucousi membrane, from one of which there had been? profuse bleeding.

One patient had a chronic gastric ulcer in thap part of the oesophagus lined by gastric mucous 
membrane, and this had not only caused stenosis but had penetrated the oesophageal wall to produce a subacute perforation into the mediastinum.

One patient developed dysphagia from a gastric carcinoma arising in, and confined to, the abnormal segment.

Some points in the clinical, radiological, and oesophagoscopic diagnosis are discussed, and the treatment carried out for this series of patients is described.
A review of the previous patients attending the clinic with peptic stenosis of the oesophagus shows that in 115 the stricture was in the squamous part of the oesophagus, and in 10 in the gastric lining. In 23 the exact pathology could not be stated with certainty.

\section{REFERENCES}

Barrett, N. R. (1950). Brit. J. Surg., 38, 175.

Bosher, L. H., and Taylor, F. H. (1951). J. thorac. Surg., 21, 306. 